\title{
Influência da temperatura e do fluxo de ar sobre o consumo de ração e ganho de peso em ratos Wistar (Rattus norvegicus) mantidos em sistema microambiental
}

\author{
Alexandre MARTINEWSKI ${ }^{1}$ \\ Nívea Lopes de SOUZA ${ }^{1}$ \\ José Luiz Bernardino \\ MERUSSE
}

\begin{abstract}
Correspondência para:
Alexandre Martinewski, Av. Prof. Orlando Marques de Paiva, $\mathrm{n}^{\circ} 87,05508-000$ Cidade Universitária, São Paulo, SP, Brasil tel (11) 3091-1408, Fax (11) 3091-7829,
\end{abstract} wski@usp.br

Recebido para publicação: 15/02/2007 Aprovado para publicação: 30/10/2008

\author{
1 - Departamento de Patologia da Faculdade de Medicina Veterinária e Zootecnia \\ da Universidade de São Paulo, São Paulo-SP
}

\section{Resumo}

Ratos wistar foram mantidos individualmente, em gaiolas metabólicas de arame, sem abrigo, em sistema microambiental, sob fluxo direto de ar a $0,6 \mathrm{~m} / \mathrm{s}$, nas temperaturas de $22^{\circ}, 24^{\circ}, 26^{\circ}, 28^{\circ}$ e $30^{\circ} \mathrm{C}$. O consumo de ração e o ganho de peso foram comparados do final de 5 dias (ANOVA; Tukey-Kramer). No total, sete grupos de 10 animais cada foram comparados. Para a faixa de $22^{\circ} \mathrm{C}$ foram utilizados três grupos, sendo um grupo experimental e dois grupos controles. Um deles foi mantido em condições ambientais semelhantes a biotérios convencionais sob ventilação geral diluidora (VGD) - C1. O outro grupo controle (C2) foi mantido no interior do equipamento de ventilação microambiental, porém, sem o direcionamento de ar, simulando a VGD. Os resultados obtidos demonstram claramente que animais mantidos sob ventilação microambiental direta a $26^{\circ}, 28^{\circ}$ e $30^{\circ} \mathrm{C}$ apresentam o mesmo ganho de massa corpórea que animais do grupo C1. Os grupos de animais mantidos a $22^{\circ}$ e $24^{\circ} \mathrm{C}$, apresentaram menor ganho de massa corpórea quando comparados a C1 ( $\mathrm{p}<0,001$ e $\mathrm{p}<0,01$ respectivamente). O ganho de peso de todos os grupos experimentais, quando comparado ao $\mathrm{C} 2$, apresenta diferenças estatísticas, exceto o mantido a $30^{\circ} \mathrm{C}$ que apresentou índice de ganho de peso equivalente a $\mathrm{C} 2$. O consumo de ração de todos os grupos se manteve constante. Somente o grupo E5 apresentou uma redução no consumo de ração quando comparado aos grupos $\mathrm{C} 1 \mathrm{e}$ C2 ( $<<0,05$ para as duas comparações).

\section{Introdução}

Os avanços obtidos no campo do controle atmosférico de biotérios permitiram que fossem claramente definidos o microambiente (interior das caixas de animais) e o macroambiente (a sala onde são mantidas as caixas de animais).

A partir da década de 70, surgem relatos de sistemas os quais efetuavam a ventilação diretamente no interior das caixas de animais. ${ }^{1,2,3}$ Tais sistemas, embora diferentes em sua concepção de projeto, possuem características que permitem classificá-los sob a definição de Ventilação Microambiental (VMA). Isto permite dois tipos específicos de controle atmosférico: i) o controle atmosférico microambiental no qual, se efetuam renovações completas de ar por hora somente no ambiente onde se encontram os animais e ii) o controle atmosférico macroambiental, voltado principalmente para o conforto humano. Dessa forma, o volume de ar a ser beneficiado com condicionamento e renovações completas (microambiente) fica drasticamente reduzido. Esta redução permite que equipamentos de menor porte, quando comparados com o sistema convencional, sejam empregados. Obviamente, o ar do macroambiente também deverá ser tratado, porém, o 
beneficiamento para conforto humano não prevê renovações completas de ar por hora, apenas um pequeno aporte de ar externo para controlar os níveis de $\mathrm{O}_{2}$ e $\mathrm{CO}_{2}$ do ambiente.

Em países tropicais, de elevadas médias de temperaturas, a simples movimentação do ar facilita a retirada do calor sensível e latente dos animais, pela convecção forçada, o que propicia sensação de conforto tanto animal quanto humano. ${ }^{4,5}$

Segundo Macintyre ${ }^{4}$, a sensação de resfriamento do homem a uma velocidade do ar de $6,5 \mathrm{~m} / \mathrm{s}$ é de, aproximadamente, $8^{\circ} \mathrm{C}$. Há, porém, limites para a indução de conforto pela simples ventilação. Umidades e temperaturas muito elevadas são fatores negativos para a criação animal. Os mecanismos de facilitação das trocas térmicas nos sistemas VMA, à temperatura ambiente, permitiriam, teoricamente, que fossem aumentadas as faixas de temperatura preconizadas para biotérios conforme literatura internacional. ${ }^{6}$ Este aumento de faixa permitiria a adoção de equipamentos de menor porte e, portanto, de menor custo de implantação.

Os procedimentos acima mencionados podem contribuir para a redução dos custos de um biotério, permitindo inclusive a adoção de equipamentos condicionadores de ar do tipo doméstico de janela para o macroambiente. Quanto ao microambiente, o processo de refrigeração foi realizado concomitantemente com um sistema VMA o que permitiu o aproveitamento do fenômeno de convecção forçada de ar sobre os animais. Este fenômeno convectivo é mais eficiente que a convecção natural, e ocorre somente quando existe a indução de um vetor de velocidade de ar sobre um corpo aquecido. ${ }^{7}$ Este fenômeno permituu trabalhar, experimentalmente, com temperaturas de conforto para os animais acima daquelas citadas na literatura para biotérios, diminuindo, desta forma, as exigências frigorígenas do equipamento. $\mathrm{O}$ condicionador de ar para o microambiente deverá, desta forma, apenas remover a carga térmica gerada pelos animais.
Com a passagem de ar diretamente pelos animais, em virtude do aumento da sensação de frio, causado pela convecção forçada, os animais apresentam variações nos índices referentes ao ganho de peso e consumo de ração, devido a mudanças no nível metabólico animal para garantir a sua homeostasia. Desta forma, torna-se necessária, portanto, uma avaliação termohigrométrica, do ambiente onde estão alojados os animais, para se avaliar qual temperatura do ar de insuflação é ideal para a manutenção destes animais sob ventilação direta. Para tanto é necessária a manutenção de animais sob condições termohigrométricas rigorosas com temperaturas distintas e constantes e a comparação do ganho de peso e consumo de ração destes animais.

\section{Material e Método}

Foram utilizados 70 ratos Wistar (Rattus norvegicus), padrão sanitário convencional controlado, machos com 45 dias de idade, provenientes do biotério do Departamento de Patologia - FMVZ/USP. Os animais foram distribuídos em sete grupos sendo dois grupos controle e cinco experimentais. Os grupos experimentais E1, E2, E3, E4 e E5 foram mantidos, sob temperaturas de $22^{\circ}, 24^{\circ}, 26^{\circ}, 28^{\circ}$ e $30^{\circ} \mathrm{C}$, respectivamente, com variação máxima de $\pm 0.1^{\circ} \mathrm{C}$. Os dois grupos controle, $\mathrm{C} 1$ e $\mathrm{C} 2$, foram mantidos a $22^{\circ} \mathrm{C}$ sendo que $\mathrm{C} 1$ foi mantido sob condições ambientais semelhantes a biotérios convencionais sob ventilação geral diluidora (VGD $222^{\circ} \mathrm{C}$ ) e C2 foi mantido no interior do equipamento de ventilação microambiental sem o direcionamento de ar, simulando a VGD a $22 \pm 0.1{ }^{\circ} \mathrm{C}$.

Tendo em vista o fato de que nenhuma inoculação experimental foi realizada e que os animais não foram submetidos a qualquer situação estressante, os mesmos puderam ser fornecidos a outros pesquisadores posteriormente.

Foi utilizada ração comercial, para ratos e camundongos, marca NUVITAL ${ }^{\circledR}$. 
No sentido de minimizar eventuais influências de diferentes formulações, foi fornecida para os grupos somente ração proveniente do mesmo lote. Os animais de ambos os grupos, experimental e controle, receberam água ad libtum.

Os resultados obtidos foram analisados comparando-se as médias obtidas dos grupos controles e experimentais. O Teste ANOVA Tukey-Kramer foi utilizado para verificar as diferenças entre o consumo de ração e o ganho de peso dos diferentes grupos.

O sistema de condicionamento de ar utilizado foi gerenciado eletronicamente via controlador eletrônico digital microprocessado $\left(\mathrm{COEL}^{\circledR}\right)$, o qual modulava a corrente elétrica de alimentação através de contatores (Telemecanique $^{\circledR}$ ), o que permitiu variação máxima de $\pm 0.1{ }^{\circ} \mathrm{C}$ da temperatura experimental. As gaiolas metabólicas foram mantidas no interior de uma caixa de isolamento térmico (CIT) em madeira (W/m ${ }^{0} \mathrm{C} @$ 0,17), com capacidade para 5 gaiolas metabólicas (Marca Beiramar - Modelo MA $121^{\circledR}$ ) com direcionamento direto do ar sobre as gaiolas dos animais. Esta passagem de ar forçada sobre os animais, provoca uma troca de calor mais intensa entre o animal e o ambiente.
Em cada gaiola metabólica foi alojado um animal. Estas gaiolas permitem controlar a quantidade de ração e água consumidas e coletar, separadamente, as fezes e a urina provenientes do metabolismo animal.

Para cada temperatura foram separados 2 lotes, de 5 animais cada, mantidos na CIT por 5 dias. Ao final do $10^{\circ}$ dia, a temperatura era modificada para a próxima temperatura experimental.

O grupo C1 foi mantido em sala com condicionamento de ar semelhante ao empregado em biotério. A temperatura deste grupo foi mantida a $22 \pm 2{ }^{\circ} \mathrm{C}$, como prevista em literatura ${ }^{8}$.

O grupo $\mathrm{C} 2$ foi mantido no interior da CIT a $22 \pm 0.1{ }^{\circ} \mathrm{C}$ mas sem o direcionamento de ar sob as gaiolas, mantendo as trocas de calor semelhantes as encontradas em biotérios convencionais.

A gaiola empregada foi do tipo metabólica, sob ventilação microambiental, no sentido de permitir um controle exato da quantidade de alimento ingerido pelo animal bem como registrar os seus excretas.

\section{Resultados}

A tabela 1 mostra os dados relativos

Tabela 1 - Consumo de ração (em gramas) por animal durante cinco dias, mantidos sob sete diferentes condições termo-higrométricas, São Paulo - 2006

\begin{tabular}{cccccccc}
\hline \multirow{2}{*}{ Animal } & \multicolumn{7}{c}{ Grupos } \\
\cline { 2 - 8 } & C1 & C2 & E1 & E2 & E3 & E4 & E5 \\
\hline 1 & 87,8 & 91,1 & 96,4 & 89,7 & 92,2 & 93,8 & 87,4 \\
2 & 94,8 & 83,1 & 85,4 & 98,4 & 84,4 & 84,6 & 84,1 \\
3 & 92,5 & 84,1 & 85,4 & 87,3 & 78,7 & 93,5 & 84,1 \\
4 & 83,6 & 90,7 & 84 & 95,3 & 82 & 95,4 & 85,1 \\
5 & 91,5 & 84,3 & 97 & 89 & 92,6 & 85,5 & 78,5 \\
6 & 90,4 & 90,6 & 96,2 & 90,3 & 81,1 & 93,5 & 73,3 \\
7 & 83,3 & 94,4 & 89,9 & 89,3 & 88,2 & 83,6 & 88,4 \\
8 & 92,7 & 95,7 & 94,8 & 90 & 74,8 & 77,3 & 73,4 \\
9 & 83,6 & 80,1 & 84,4 & 99,8 & 81,4 & 78,4 & 79,9 \\
10 & 95,2 & 96,5 & 101,2 & 88,8 & 74,7 & 85,2 & 77,9 \\
\hline Média & $89,54{ }^{(1)}$ & $89,06{ }^{(2)}$ & $91,47^{(3)}$ & $91,79{ }^{(4)}$ & 83,01 & 87,08 & 81,21 \\
\hline Desvio-padrão & 4,66 & 5,78 & 6,37 & 4,38 & 6,39 & 6,60 & 5,43 \\
\hline
\end{tabular}

(1) - Diferença estatisticamente significativa com o E5 $(p<0,05)$

(2) - Diferença estatisticamente significativa com $\circ$ E5 $(p<0,05)$

(3) - Diferença estatisticamente significativa com o E3 $(p<0,05)$ e com o E5 $(p<0,01)$

(4) - Diferença estatisticamente significativa com $\circ$ E3 $(p<0,05)$ e com $\circ$ E5 $(p<0,01)$ 
ao consumo de ração, em gramas, pelos ratos no decorrer de cinco dias em sete diferentes condições de ventilação e temperatura. Foram notadas diferenças estatisticamente significantes no consumo de ração entre os grupos, o grupo $\mathrm{C} 1$ $\left(22 \pm 2^{\circ} \mathrm{C}\right)$ e $\mathrm{C} 2\left(22 \pm 0,1^{\circ} \mathrm{C}\right)$ consumiram mais ração que o grupo $\mathrm{E} 5\left(30 \pm 0,1^{\circ} \mathrm{C}\right)$. O Grupo E1 $\left(22 \pm 0.1^{\circ} \mathrm{C}\right)$ consumiu mais ração que E3 $\left(26 \pm 0.1^{\circ} \mathrm{C}\right)$ e E5 $(30 \pm$ $0.1^{\circ} \mathrm{C}$ ) e o grupo E2 mais ração que o E3 e E5.

A tabela 2 mostra o ganho de peso, em gramas, dos ratos mantidos em sete diferentes condições de ventilação e temperatura, no decorrer de cinco dias. Quando os grupos foram comparados estatisticamente o ganho de peso do grupo $\mathrm{C} 1\left(22 \pm 2^{\circ} \mathrm{C}\right)$ foi maior dos grupos $\mathrm{E} 1\left(22 \pm 0,1^{\circ} \mathrm{C}\right)$ e E2 $\left(24 \pm 0,1^{\circ} \mathrm{C}\right)$. $\mathrm{O}$ grupo $\mathrm{C} 2$ ganhou mais peso que os grupos E1, E2, E3 $\left(26 \pm 0,1^{\circ} \mathrm{C}\right)$ e $\mathrm{E} 4$ $\left(28 \pm 0,1^{\circ} \mathrm{C}\right)$. O grupo E1 teve um aumento de peso menor que E3, E4 e E5 $\left(30 \pm 0,1^{\circ} \mathrm{C}\right)$. O grupo $\mathrm{E} 2$ ganhou menos peso que E4 e E5 e o grupo E3 menos que E5.

\section{Discussão}

Pode-se deduzir que com o aumento da temperatura, os animais tendem a consumir menos ração devido ao menor consumo calórico para a manutenção do seu metabolismo.

Tendo em vista que o grupo controle foi mantido sob condições convencionais de condicionamento térmico, compreendido como o ambiente do laboratório, no qual o experimento foi conduzido, $22 \pm 2^{\circ} \mathrm{C}$, foi necessária a repetição das condições do grupo controle, agora, porém, no interior da CIT, sem direcionamento de ar sobre os animais, simulado-se uma condição de ventilação geral diluidora, na qual poderíamos obter um ambiente com temperatura de $22 \pm 0,1^{\circ} \mathrm{C}$.

Estes dados mostram que quanto maior a temperatura do fluxo de ar, direcionado para os animais, maior o ganho de peso dos grupos. Este aumento do ganho de peso tende a se igualar aos grupos controle na faixa de 28 a $30^{\circ} \mathrm{C}$.

Ficou evidente uma relação linear direta entre o ganho de peso e o aumento

Tabela 2 - Ganho de peso (em gramas) por animal durante cinco dias, mantidos sob sete diferentes condições termo-higrométricas, São Paulo - 2006

\begin{tabular}{|c|c|c|c|c|c|c|c|}
\hline \multirow{2}{*}{ Animal } & \multicolumn{7}{|c|}{ Grupos } \\
\hline & $\mathrm{C} 1$ & $\mathrm{C} 2$ & E1 & $\mathrm{E} 2$ & E3 & E4 & E5 \\
\hline 1 & 22,6 & 20,4 & 19,6 & 17,4 & 22,4 & 21,7 & 21,2 \\
\hline 2 & 22 & 21 & 14 & 17,2 & 22 & 20,8 & 23,5 \\
\hline 3 & 18,6 & 21,6 & 16 & 15,6 & 21,6 & 21,4 & 19,8 \\
\hline 4 & 18,9 & 20,2 & 15,1 & 17,6 & 20,8 & 21,5 & 19,7 \\
\hline 5 & 20,6 & 22,6 & 16,8 & 14,5 & 19,6 & 22 & 22,2 \\
\hline 6 & 20,1 & 24,6 & 13,6 & 15,4 & 19,3 & 17,5 & 24,4 \\
\hline 7 & 20,6 & 24 & 13,8 & 17,2 & 16,6 & 19,2 & 24,4 \\
\hline 8 & 22,2 & 23,3 & 16,8 & 19,6 & 16,2 & 18,8 & 22,2 \\
\hline 9 & 22,2 & 24,8 & 14,7 & 19,4 & 17 & 17,2 & 20,6 \\
\hline 10 & 20,4 & 23,6 & 13 & 17,9 & 16,3 & 17,8 & 21,1 \\
\hline Média & $20,82^{(1)}$ & $22,61^{(2)}$ & $15,34(3)$ & $17,18^{(4)}$ & $19,18^{(5)}$ & 19,79 & 21,91 \\
\hline Desvio-padrão & 1,41 & 1,71 & 2,00 & 1,65 & 2,48 & 1,89 & 1,74 \\
\hline \multicolumn{8}{|c|}{$\begin{array}{l}\text { (1) - Diferença estatisticamente significativa com oE1 }(p<0,001) \text { e com E2 }(p<0,001) \\
\text { (2) - Diferença estatisticamente significativa com oE1 }(p<0,001) \text {, com o E2 }(p<0,001) \text {, com o E3 }(p<0,01) \text { e c } \\
\text { (p<0,05) } \\
\text { (3) - Diferença estatisticamente significativa com oE3 }(p<0,001) \text {, com o E4 }(p<0,001) \text { e com oE5 }(p<0,001) \\
\text { (4) - Diferença estatisticamente significativa com o E4 }(p<0,05) \text { e com o E5 }(p<0,001) \\
\text { (5) - Diferença estatisticamente significativa com oE5 }(p<0,05)\end{array}$} \\
\hline
\end{tabular}


da temperatura a que os diferentes grupos foram submetidos. Isto indica maior conversão, da ração consumida, para a massa corpórea em temperaturas mais elevadas e maior conversão para energia em temperaturas mais baixas, o que poderia ser explicado pela necessidade da manutenção de homeostasia do animal. Lembramos que em temperaturas mais baixas de ar e aplicando-se a ele uma certa velocidade, a sensação térmica de maior frio se intensifica.

Os fatos comentados até então levanos a questionar fortemente a literatura sobre condicionamento de ar para biotérios produzida até o momento. Sob condições de ventilação microambiental, existe uma forte influência do fator "convecção forçada" sobre os animais. Tal fenômeno permite que se obtenha, nestes, sensação térmica de conforto com temperaturas de ar maiores do que as citadas pela literatura mundial para sistemas convencionais. Lembramos que mesmo sem qualquer rebaixamento da temperatura de bulbo seco de determinada massa de ar, somente o deslocamento dessa massa, sobre um organismo (convecção forçada), já é capaz de remover energia térmica, provocando uma sensação de resfriamento que poderá chegar ate o desconforto na dependência da velocidade dessa massa de ar, da umidade presente e, obviamente, da temperatura dessa massa de ar.

Com relação ao conforto humano, esse assunto já foi suficientemente abordado, existindo, inclusive, diversos tipos de cartas de conforto térmico, as quais podem facilmente ser consultadas pelos técnicos para projetos de sistemas de condicionamento de ar para conforto humano. Com relação a animais, em especial os roedores de laboratório, estudos deste tipo merecem ainda muita demanda em trabalhos experimentais que levem em consideração as diferentes espécies, bem como as distintas condições fisiológicas.

Os grupos experimentais mantidos sob diferentes temperaturas mostraram umidade mais baixa nas sobras de ração, quando comparada ao grupo controle. Fato semelhante foi descrito por Carissimi et al. ${ }^{9}$, com relação à maravalha em sistemas microambientais, onde a constante passagem de ar sobre os animais e conseqüentemente sobre maravalha diminui drasticamente o teor de umidade desta, o que poderia contribuir para a menor geração de vapores de amônia.

\section{Agradecimento}

À Fundação de Amparo à Pesquisa do Estado de São Paulo, processo no ${ }^{\circ}$ 02/ 09908-0, pelo suporte financeiro.

\section{Influence of temperature and air flow in the food intake and weigth gain in Wistar rats (Rattus norvegicus) kept on microenvironmental system for laboratory animals.}

\begin{abstract}
Wistar rats had been kept individually, in metabolic wire cages, without shelter, in microenvironmental system, under direct air flow at 0,6 $\mathrm{m} / \mathrm{s}$, under temperatures of $22^{\circ}, 24^{\circ}, 26^{\circ}, 28^{\circ}$ and $30^{\circ} \mathrm{C}$. The food consumption and the weight gain had been compared in the end of 5 days (ANOVA; Tukey-Kramer). In the total, 7 groups, 10 animals each, had been compared. For the $22^{\circ} \mathrm{C}$ temperature, had been used 3 groups, one experimental and two controls. One of them was kept in similar ambient of conventional laboratory animal rooms conditions (general diluitory ventilation, GDV) - C1. The other control group (C2) was kept in the interior of the equipment of microenvironmental ventilation, however, without the direct air flow, simulating the GDV. The gotten results demonstrate clearly that animal
\end{abstract}

Key words:

Laboratory Animals. Rats.

Weight gain. Microenvironment. 
kept under direct microenvironmental ventilation at $26^{\circ}, 28^{\circ}$ and $30^{\circ} \mathrm{C}$ have the same gain of corporal mass that $\mathrm{C} 1$ group. The groups kept at $22^{\circ}$ and $24^{\circ} \mathrm{C}$, had less corporal mass gain when compared to $\mathrm{C} 1$ $(\mathrm{p}<0,001$ and $\mathrm{p}<0,01$ respectively). The weight gain for all the experimental groups, when compared to $\mathrm{C} 2$, presents statistical differences, except $30^{\circ} \mathrm{C}$ group, that was equal to $\mathrm{C} 2$. The food consumption of all the groups was constant. Only the $30^{\circ} \mathrm{C}$ group presented a reduction in the food consumption when compared with the groups $\mathrm{C} 1$ and $\mathrm{C} 2$ ( $\mathrm{p}<0,05$ for the two comparisons).

\section{Referências}

1 GAMBLE, M. R.; CLOUGH, G. Ammonia build-up in animal boxes and its effect on rat tracheal epithelium. Laboratory Animals, v. 10, n. 2, p. 93-104, 1976.

2 GORTON, R. L. System design and energy conservation considerations. ASHRAE Transactions: symposia, v. 81, 2nd part, p. 572-578, 1975.

3 GORTON, R. L.; WOODS, J. E.; BESCH, E. L. System load characteristics and estimation of annual heat loads for laboratory animal facilities. ASHRAE Transactions: symposia, v. 82, 1st part, p. 107-112, 1976.

4 MACINTYRE, A. J. Ventilação industrial e controle da poluição. Rio de Janeiro: Guanabara Koogan, 1990. p. 1-3, 26-36, 92-104.

5 MESQUITA, A. L. S.; GUIMARÃES, F. A.; NEFUSSI, N. Engenharia de ventilação industrial. São Paulo: CETESB, 1988. p. 121-188.

6 SOCIETY FOR LABORATORY ANIMAL SCIENCE. Committe on training and correct housing of laboratory animals. On the planning and structure of animal facilities for institues performing animal experiments. 2 ed. Basel: Solas, 1989. p. 39-48.

7 HOLMAN J. P. Heat transfer. New York: MacGrawHill Inc., 1981.

8 CANADIAN COUNCIL ON ANIMAL CARE. Guide to the care and use of experimental animals. Ontario, CCAC, 1984. v.2.

9 CARISSIMI, A. S.; CHAGURI, L. C.; TEIXEIRA, M. A.; MORI, C. M. C.; MACCHIONE, M.; SANT'ANNA, E. T. G.; SALDIVA, P. H. N.; SOUZA, N. L.; MERUSSE, J. L. B. Effects of two ventilation systems and bedding change frequency on cage environmental factors in rats (Rattus norvegicus). Animal Technology , v. 51 , n. 3 , p. $161-170,2000$. 